\title{
Manejo artroscópico de fractura de espina tibial con TightRope en paciente pediátrico
}

\author{
Arthroscopic management of tibial spine fracture \\ with TightRope in pediatric patient
}

\author{
René Ochoa Cázares, * René Gabriel Narváez Corona ${ }^{\ddagger}$ \\ Citar como: Ochoa CR, Narváez CRG. Manejo artroscópico de fractura de espina tibial con TightRope en paciente \\ pediátrico. Acta Med Grupo Angeles. 2021; 19 (2): 262-266. https://dx.doi.org/10.35366/100452
}

Resumen

Las fracturas de espina tibial en pacientes pediátricos representan un reto para su manejo quirúrgico debido al riesgo de lesionar el fragmento o la fisis de crecimiento. Se han desarrollado múltiples técnicas de manejo, entre las cuales la vía artroscópica continúa siendo la mejor opción para permitir una recuperación más rápida, la preservación o la mayor conservación de la fisis de crecimiento posible sin la necesidad de una segunda intervención que requiera retiro de material. El uso de TightRope proximal y distal permite una reducción óptima con preservación del fragmento de la espina tibial así como un túnel pequeño y único a través de la fisis que no compromete el crecimiento.

Palabras clave: Espina tibial, artroscopia, TightRope.

\section{INTRODUCCIÓN}

Las fracturas de espina tibial pueden producir una rodilla inestable. Su fijación puede complicarse posteriormente con arresto fisario. Las fracturas son más comunes en niños que en niñas y ocurren con una incidencia pico entre los ocho y 14 años de edad. Las lesiones a nivel de rodilla

* Director del Curso Artroscopia y Reconstrucción Articular.

‡ Fellow en Artroscopia y Reconstrucción Articular.

Hospital Ángeles Pedregal, Facultad Mexicana de Medicina Universidad La Salle Campus México.

Correspondencia:

Dr. René Gabriel Narváez Corona

Correo electrónico: narvaezrg87@gmail.com

Aceptado: 01-05-2020.

www.medigraphic.com/actamedica

\section{Abstract}

Fractures of the tibial spine in pediatric patients are a challenge for surgical treatment because of risk of damage to the physis and the fragment itself. There have been different techniques in development, from which arthroscopic treatment remains the best option for faster rehabilitation, as well as preservation of the physis or the least possible disruption of it, and no need for a second procedure for implant removal. The use of TightRope system in both ends of the fixation allows for optimal reduction with preservation of the tibial spine fragment and the physis with little to no growth compromise.

Keywords: Tibial spine, arthroscopy, TightRope.

son relativamente poco comunes, y si su manejo no es el adecuado, pueden producir complicaciones importantes debido a que la mayor parte del crecimiento de la extremidad inferior ocurre a nivel de la rodilla, correspondiendo la tibia a $55 \%$ del crecimiento de la extremidad. ${ }^{1,2}$ Se presenta el caso de paciente pediátrico con fractura de espina tibial manejado con reducción por vía artroscópica y el sistema TightRope.

\section{CASO CLÍNICO}

Paciente masculino de 11 años que acude a valoración tras presentar caída mientras jugaba en un brincolín, con lo que desarrolla dolor y aumento de volumen de rodilla derecha así como equimosis leve y limitación para el apoyo con la extremidad. Se realizan estudios de imagen (Figuras 1 y 2), entre ellos radiografía de ambas rodillas en la que se describe irregularidad de la meseta tibial de rodilla derecha, compatible con avulsión de la espina tibial. La tomografía computarizada mostró fractura de meseta tibial 
en topografía de inserción de ligamento cruzado anterior. La resonancia magnética reporta fractura por avulsión de la apófisis espinosa anterior de la tibia, y zonas de contusión ósea con lesión osteocondral grado 1-2 de cóndilo femoral lateral y plataforma tibial, además de meniscopatía intrasustancia medial grado 2 .

\section{Técnica quirúrgica}

Se programó para reducción de meseta tibial por vía artroscópica mediante sistema TightRope, para lo que se coloca al paciente en decúbito supino, con rodilla a $90^{\circ}$ de flexión y sujetador, se realizan portales anteromedial y anterolateral de rodilla derecha, con lo que se vacía hemartrosis de rodilla derecha, limpiando el foco de fractura. Se procede a realizar incisión en tibia proximal en superficie anteromedial para perforar túnel tibial mediante colocación de guía para ligamento cruzado anterior a $55^{\circ}$, se perfora con broca $3.5 \mathrm{~mm}$ a través de fragmento de espina tibial reducido con la misma guía, introduciendo desde túnel tibial un FiberStick con sutura de FiberWire para recuperar suturas de TightRope. Se extraen cabos de sutura a través de túnel tibial y se pasa TightRope a través de portal anteromedial, con las suturas atravesando fragmento de espina tibial. Se gira TightRope a su posición horizontal y se tensa con cuidado de no dañar el fragmento de espina tibial (Figuras 3 y 4), se fija en pared anteromedial de tibia proximal con otro TightRope distal circular anudando suturas, y se cortan excesos de éstas. Se cierran portales e incisión de tibia proximal, se cubre con vendaje de Jones y se coloca rodillera mecánica graduada a $0^{\circ}$ de extensión. En consultas subsecuentes se fue ampliando el arco de movilidad de rodilla a $45^{\circ}$ de flexión y se verificó con control radiográfico la consolidación del foco de fractura. Comienza con apoyo parcial y continúa con fisioterapia en casa cuatro semanas después de la cirugía, ampliando el arco de movimiento a $90^{\circ}$ de flexión.

\section{DISCUSIÓN}

Las fracturas de espina tibial ocurren por lo general entre los ocho y 14 años de edad. Son lesiones raras con incidencia de 3/100,000 niños por año. Aproximadamente 5\% de las lesiones que producen derrame articular en rodilla son por fractura de espina tibial. ${ }^{1,2}$ Esta lesión se ha descrito también entre 2 y $5 \%$ de las lesiones de rodilla y $14 \%$ de las lesiones de ligamento cruzado anterior en pacientes con esqueleto inmaduro. ${ }^{3,4}$

La fisis de crecimiento a nivel de la rodilla aporta 55\% del crecimiento a la estatura total del paciente. La tibia proximal aporta un crecimiento de $6 \mathrm{~mm}$ por año hasta la edad de 13 años. La fusión de la tuberosidad tibial a los 16 años en mujeres y a los 18 años en hombres representa la madurez ósea final de miembros inferiores. ${ }^{2,3}$

El mecanismo de lesión, de acuerdo con la evidencia más reciente, sugiere que la lesión ocurre fuera de actividades deportivas, aunque también se ha descrito que la lesión puede presentarse por mecanismo de hiperextensión de la
Figura 1:

A) Rayos X lateral de rodilla derecha con irregularidad de la meseta tibial. B) Resonancia magnética nuclear con ruptura completa del ligamento cruzado anterior con fractura por avulsión de la apófisis espinosa de la tibia con discontinuidad

de la bisagra posterior y desplazamiento hacia posterior del fragmento.
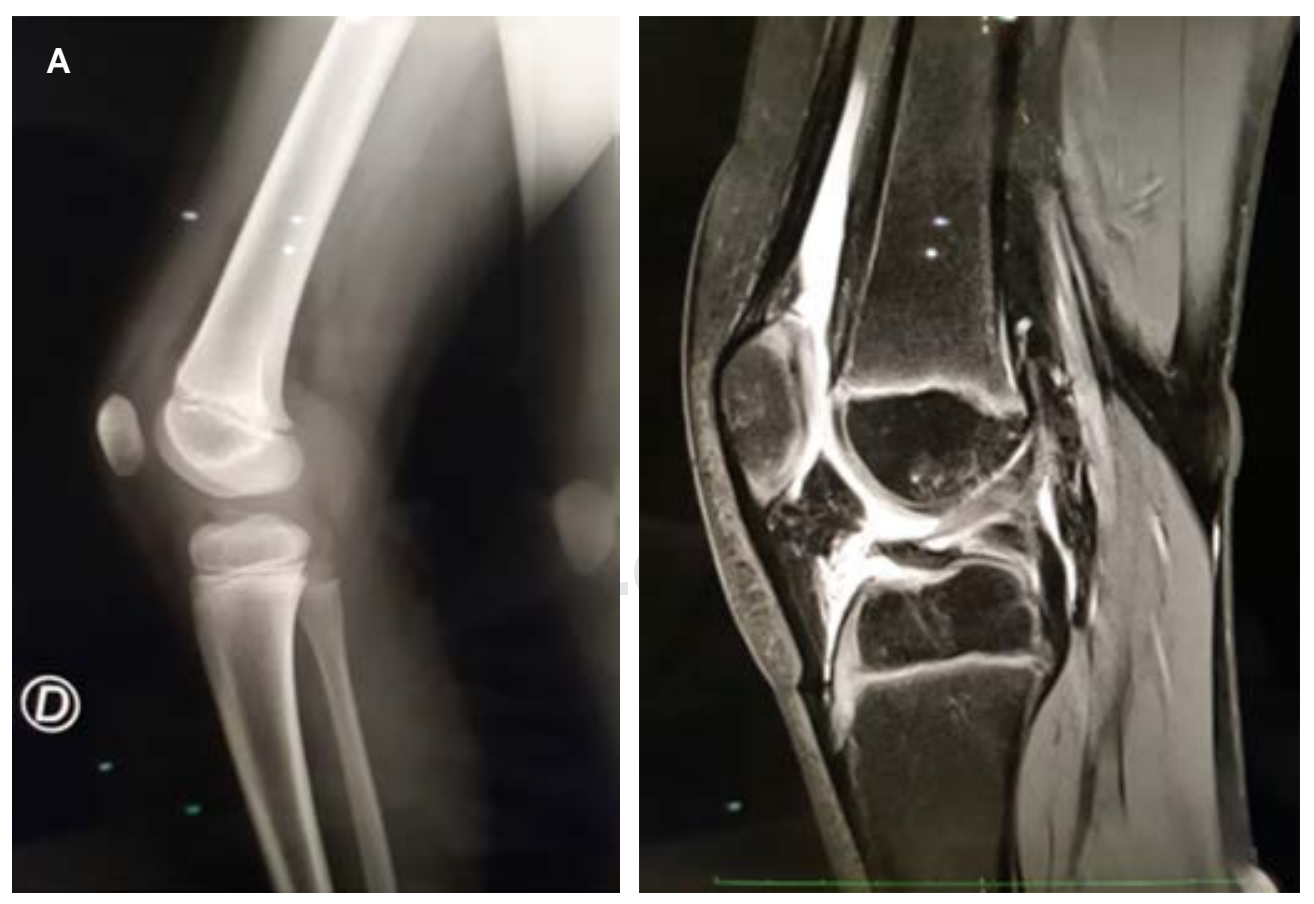
Figura 2:

A) Foco de fractura de la espina tibial con fragmentos

libres. B) Una vez que

se limpia, se perfora con broca $3.5 \mathrm{~mm}$, se introduce

FiberStick con FiberWire para recuperar las suturas del sistema TightRope.
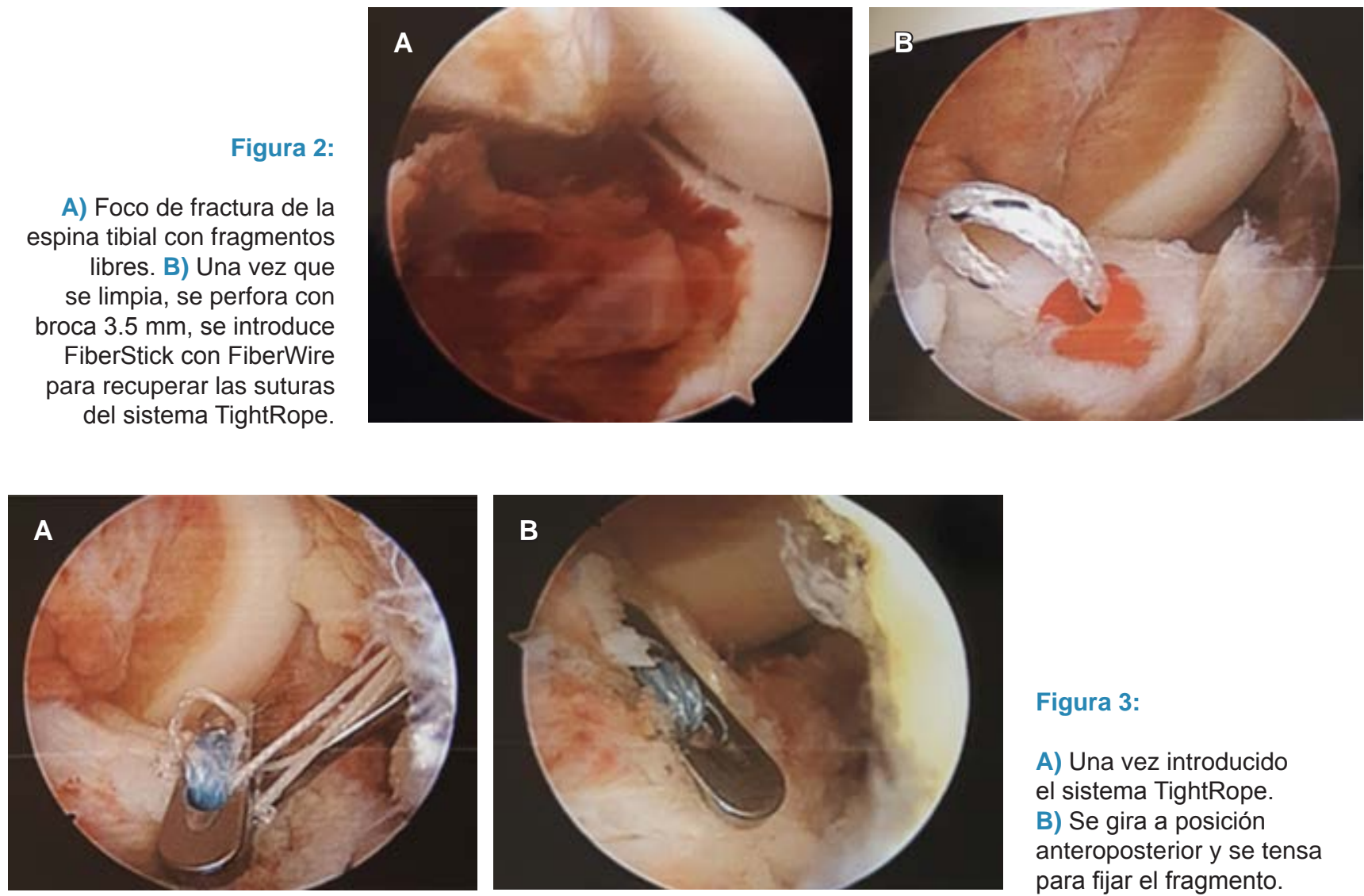

Figura 3:

A) Una vez introducido el sistema TightRope.

B) Se gira a posición anteroposterior y se tensa para fijar el fragmento.

rodilla con rotación, como puede ocurrir en el balompié, tras una caída de bicicleta u otras actividades de contacto. La osificación incompleta de esta zona vuelve la espina tibial más débil que el ligamento cruzado anterior, por lo que el mecanismo de pivoteo que produce ruptura de ligamento cruzado anterior puede producir avulsión de la espina tibial. 1,3,4

En un paciente pediátrico con antecedente traumático deben tomarse radiografías para descartar una fractura de espina tibial. Una vez que se descarta puede procederse a una exploración de la rodilla. ${ }^{5}$

La clasificación de estas lesiones está basada en el grado de desplazamiento en la radiografía lateral. Meyers y McKeever las clasifican en tres tipos. Las fracturas tipo 1 no están desplazadas; las fracturas tipo 2 están desplazadas, pero conservan una bisagra posterior intacta; las fracturas tipo 3 están completamente desplazadas. Un cuarto tipo de fractura se usa para describir un patrón con conminución de la espina tibial. ${ }^{1}$ De acuerdo con tal clasificación, al paciente correspondió una fractura de espina tibial tipo 3. La complementación diagnóstica con resonancia magnética adquiere importancia ante la sospecha de fracturas de espina tibial tipo 1. Se ha encontrado una baja sensibilidad para detectar rupturas parciales de ligamento cruzado anterior con este estudio. ${ }^{5}$ Además, la resonancia magnética puede facilitar la planeación preoperatoria al definir de manera más adecuada la topografía de la avulsión así como lesiones adicionales de tejidos blandos que requieran manejo. Pueden existir lesiones asociadas en $40 \%$ de los casos. Hasta $35 \%$ de los casos reportan atrapamiento meniscal en el sitio de fractura, y contusiones óseas en 90\%. ${ }^{1}$

Las fracturas tipo 1 pueden resolverse con manejo conservador, mientras que las fracturas tipo 2 y 3 que pueden reducirse exitosamente bajo anestesia, pueden manejarse de forma conservadora. Se recomienda inmovilización entre $10-20^{\circ}$ de flexión, donde el ligamento cruzado anterior se encuentra bajo menor tensión. ${ }^{1,5}$ Las fracturas tipo 2 y 3 que no pueden reducirse y las lesiones tipo 4 son quirúrgicas. Se ha observado que en muchas ocasiones el cuerno anterior del menisco medial evita una reducción adecuada. ${ }^{1}$

Se han descrito técnicas artroscópicas y abiertas para la reducción y fijación de fracturas de espina tibial. La reducción artroscópica y fijación interna ha mostrado tasa baja de morbilidad, permite movilización más rápida y menor estancia hospitalaria en comparación con reducción abierta. 

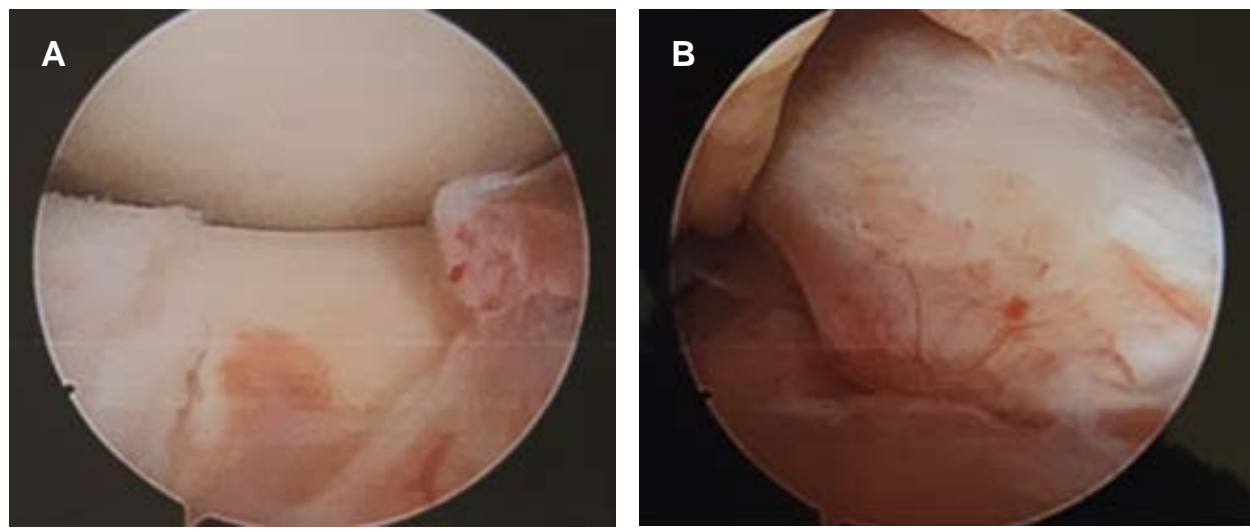

A) Reducción del fragmento al término del procedimiento. B) Se observa tensión adecuada del ligamento cruzado anterior.

Puede emplearse reducción con un tornillo canulado anterógrado o con técnicas de anclaje a través de un túnel tibial utilizando una guía para ligamento cruzado anterior, ${ }^{1}$ como fue el caso del paciente.

El manejo quirúrgico puede dividirse en técnicas con o sin preservación fisaria. El manejo con técnica transfisaria se ha convertido en el estándar en adultos y pacientes adolescentes. Pacientes pediátricos con fisis en crecimiento en etapa Tanner 1 y 2 han obtenido buenos resultados con técnica transfisaria entre edades de 11 y 12.1 años. Por lo general se observan alteraciones en el crecimiento por arresto fisario tras el uso de injertos hueso-tendón-hueso para reconstrucción de ligamento cruzado anterior, por lo que no se recomienda su uso. Perforaciones de $9 \mathrm{~mm}$ transtibiales producen una disrupción de $6.6 \%,{ }^{5}$ la cual disminuye cuanto más vertical sea la perforación, por lo que una perforación única de $3.5 \mathrm{~mm}$ a $55^{\circ}$ resulta insignificante en el manejo de este paciente.

Entre las técnicas descritas se encuentra el uso de clavillos de Kirshner percutáneos, fijación con tornillos guiados por artroscopia, grapas y el uso del sistema TightRope. ${ }^{3,4,6}$ Muchos de estos procedimientos requieren de una segunda intervención para el retiro de material, además de asociarse a complicaciones como fatiga del implante, aflojamiento o migración, infección y ausencia de consolidación. ${ }^{4,6}$ Se ha encontrado además en un estudio en cadáveres que la fuerza de fijación con sutura FiberWire es mayor que la de fijación con tornillos. ${ }^{4}$

Se ha descrito una técnica en la que se atraviesan las fibras distales del ligamento cruzado anterior con FiberWire o Ethibond y con fijación distal con un TightRope de cuatro agujeros contra la cortical de tibial proximal. ${ }^{3}$ Esta técnica requiere una perforación adicional para la fijación provisional con clavillo de Kirshner. Consideramos que el uso de un botón de TightRope proximal que tracciona el fragmento óseo sin involucrar las fibras del ligamento cruzado anterior, es más bondadoso con los tejidos nativos, además de que no se pasa sutura por los bordes del fragmento, con lo que puede comprometerse el contacto y la congruencia entre el fragmento de la espina tibial y el resto de la epífisis, además de requerir una perforación única de $3.5 \mathrm{~mm}$ del fragmento de la espina tibial, por lo que conserva más su integridad. También se han descrito técnicas que requieren de dos orificios a los lados del fragmento a reducir que ofrece una reducción adecuada. ${ }^{7}$ Sin embargo, preferimos mantener las perforaciones al mínimo para dañar lo menos posible la fisis de crecimiento.

Las principales complicaciones son laxitud, artrofibrosis y ausencia de consolidación. Las fibras del ligamento cruzado anterior pueden distenderse antes de que se presente una fractura de la espina tibial, ${ }^{4}$ por lo que a pesar de un manejo adecuado puede desarrollarse laxitud hasta en $60 \%$ de los pacientes con fracturas tipo 3 o $4 .^{1}$ A tres meses de tratamiento no se han encontrado datos sugestivos de laxitud en el paciente de este trabajo. La tasa de artrofibrosis ocurre alrededor de 10\%, y se asocia a un retraso en la movilización postquirúrgica de más de cuatro semanas. ${ }^{1}$

\section{CONCLUSIÓN}

El riesgo de inestabilidad de rodilla en pacientes pediátricos amerita un manejo adecuado cuidando no comprometer el crecimiento debido a una lesión fisaria. El riesgo de presentar laxitud, a pesar de un manejo adecuado, debe ser consultado con los padres. Las técnicas de reducción artroscópica han permitido una recuperación más temprana y se continúan desarrollando técnicas de preservación fisaria para no comprometer el crecimiento.

\section{REFERENCIAS}

1. Bailey MEA, Wei R, Bolton MS, Richards, RH. Paediatric injuries around the knee: bony injuries. Injury. 2019; 51 (3): 611-619. 
2. Canale ST, Beaty JH. Fractures and dislocations in children. En: Azar FM, Canale ST, Beaty JH. Campbell's operative orthopaedics. Philadelphia, PA: Elsevier/Mosby; 2016. pp. 1479-1481.

3. Elsaid, ANS, Zein AMN, ElShafie M, El Said NS, Mahmoud AZ. Arthroscopic single-tunnel pullout suture fixation for tibial eminence avulsion fracture. Arthrosc Tech. 2018; 7 (5): e443-e452.

4. Gamboa JT, Durrant, BA, Pathare NP, Shin EC, Chen JL. Arthroscopic reduction of tibial spine avulsion: suture lever reduction technique. Arthrosc Tech. 2017; 6 (1): 121-126.
5. Diwakar M. Management of $\mathrm{ACL}$ tear in paediatric age group: a review of literature. J Arthrosc Jt Surg. 2018; 5 (1): 9-14.

6. Loriaut P, Moreau PE, Boyer P. Arthroscopic treatment of displaced tibial eminence fractures using a suspensory fixation. Indian J Orthop. 2017; 51 (2): 187-191.

7. Hara K, Kubo T, Shimizu C, Suginoshita T, Hirasawa Y. Arthroscopic reduction and fixation of avulsion fracture of the tibial attachment of the anterior cruciate ligament. Arthroscopy. 2001; 17 (9): 10031006. 